\title{
Knowledge Solutions
}

Learning is the key to success - some would even say survival - in today's organizations.

Knowledge should

be continuously enriched through both internal and external learning. For this to happen, it is necessary to support and energize organization, people, knowledge, and technology for learning.

\section{Building a Learning Organization}

\section{By Olivier Serrat}

\section{The Learning Organization Model}

For organizations wishing to remain relevant and thrive, learning better and faster is critically important. Many organizations apply quick and easy fixes often driven by technology. Most are futile attempts to create organizational change. However, organizational learning is neither possible nor sustainable without understanding what drives it. The figure below shows the subsystems of a learning organization: organization, people, knowledge, and technology. Each subsystem supports the others in magnifying the learning as it permeates across the system.
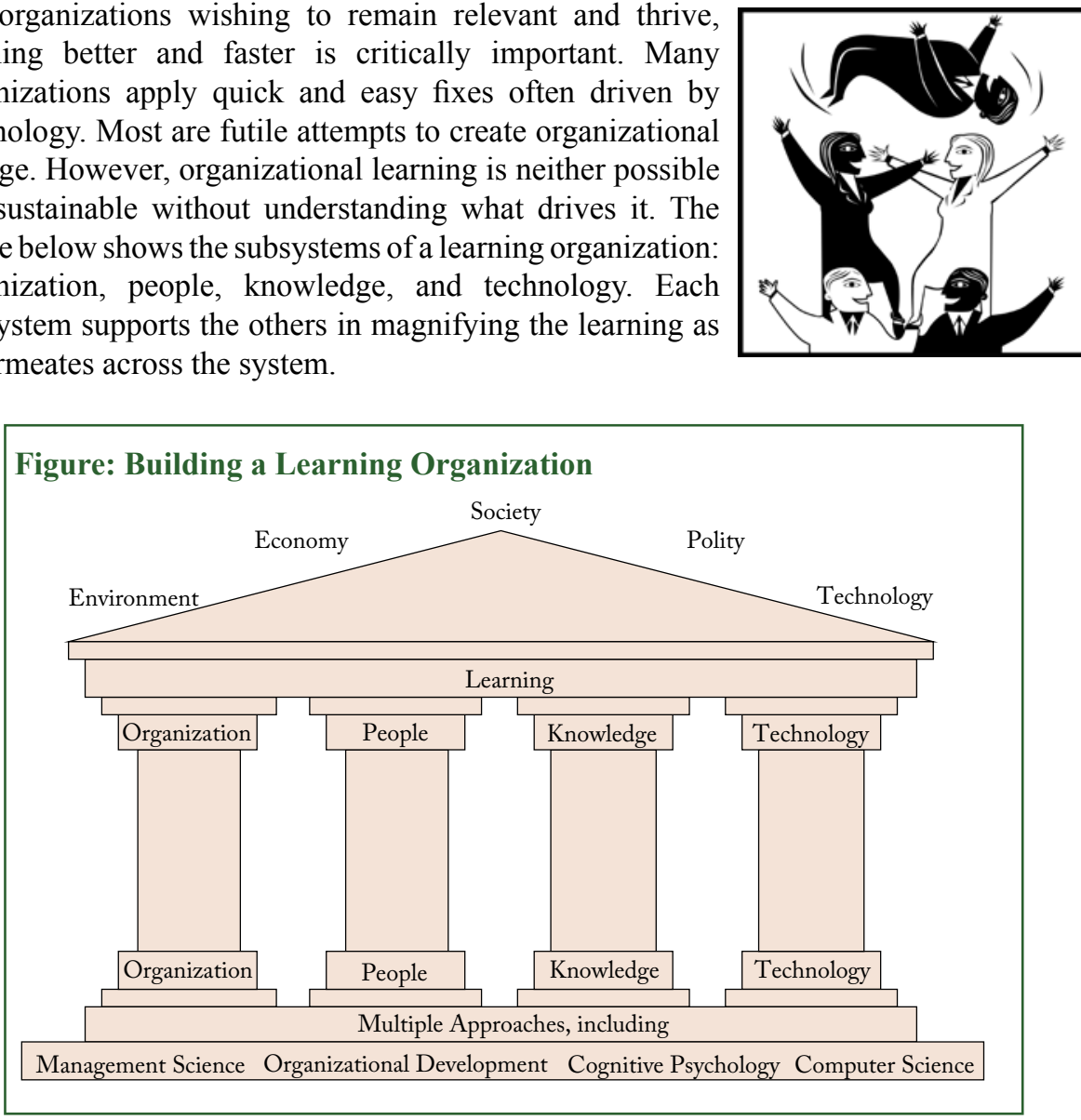

Source: Author 


\section{Organization}

A learning organization values the role that learning can play in developing organizational effectiveness. It demonstrates this by having an inspiring vision for learning and a learning strategy that will support the organization in achieving its vision.

The leadership of a learning organization is committed to the importance of learning and clearly communicates that

It is not the strongest of the species who survive, nor the most intelligent; rather it is those most responsive to change.

Charles Darwin learning is critical to organizational success. The leadership recognizes the importance of providing the motive, means, and opportunity for learning: (i) the motive being the "why?"- the purpose and reason for learning; (ii) the means being the "how and what?"- the models, methods, and competencies required; and (iii) the opportunity being the "where and when?"- the spaces for learning. Leaders take an exemplary leading role in creating and sustaining a supportive learning culture.

The supreme accomplishment is to blur the line between work and play. -Arnold Toynbee

The structure of a learning organization takes into account the common obstacles to learning so it is carefully aligned with strategy, avoiding the development of "silos" and minimizing unnecessary levels of hierarchy.

Communication systems are used to facilitate the lateral transfer of information and knowledge across formal structural boundaries. In decentralized and geographically spread organizations, particular care is taken to use communication to encourage lateral communication and to overcome the increased danger of the development of "silos".

Adequate resources are allocated for learning in terms of time, space, specialist support staff, and budgets for knowledge management and learning infrastructure, formal and informal communities of practice ${ }^{1}$ and other value networks (both internal and external), ${ }^{2}$ and learning and development programs. Support to communities of practice, for example, is extended in a structured manner throughout their life cycle. ${ }^{3}$

To stimulate creativity and generate new insights and innovative practices, a learning organization takes a balanced approach to the importance of both planned and emergent learning. Planned learning is addressed through the careful development of strategy, structure, systems, procedures, and plans. In a learning organization, planning is based on careful reflection through probing questions that draw on data and information from monitoring, review, and self- and independent evaluation.

Emergent learning is equally important but takes an inherently more speculative and opportunistic approach. It is dependent on encouraging a passion for learning and knowledge sharing among staff members, developing learning competencies, creating opportunities for informal sharing, and cultivating a supportive learning culture.

Failures and unintended outcomes are the focus of constructive discussions leading to new approaches. When such incidents involve clients, care is taken to protect their reputation.

\section{People}

A learning organization needs people who are intellectually curious about their work, who actively reflect on their experience, who develop experience-based theories of change and continuously test these in practice with colleagues, and who use their understanding and initiative to contribute to knowledge development. In short, it needs people who are

I have no special talents. I am only passionately curious. -Albert Einstein

Communities of practice emerge in the social space between project teams and knowledge networks. They are groups of like-minded, interacting people who filter, analyze, invest and provide, convene, build, and learn and facilitate to ensure more effective creation and sharing of knowledge in their domain. What they know, who they are, and what they do define them.

2 A value network is any web of relationships that generates both tangible and intangible value through complex dynamic exchanges. Value networks include communities of practice, knowledge networks, and networks of practice. Their growing importance requires that organizations pay more attention to their forms and functions, evolve principles of engagement, circumscribe and promote success factors, and monitor and evaluate performance with knowledge performance metrics.

3 The five stages of community development are potential, coalescing, maturing, stewardship, and transformation. 
reflective practitioners. Reflective practitioners understand their strengths and limitations and have a range of tools, methods, and approaches for knowledge management and learning, individually and in collaboration with others.

Reflective practice flourishes when people experience a high level of psychological safety and trust, and it is undermined when people feel exposed to unfair negative criticism and when they believe that they cannot rely on colleagues. Teamwork is, therefore, a vital ingredient of a genuine learning organization. Indeed, one characteristic of teams in learning organizations is that they operate as learning communities in which sensitively

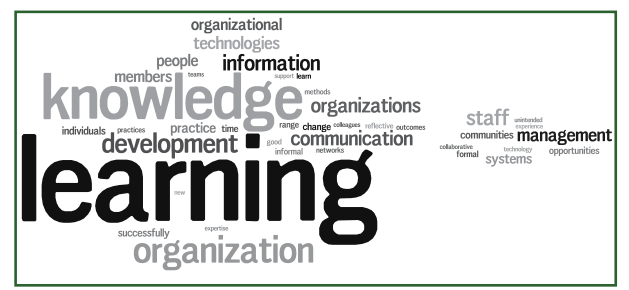
expressed dissent, conflict, and debate are encouraged as positive sources of learning. Developing the safety and trust on which reflective practice and positive teamwork depend requires careful attention to relationship building and the management of individual and collective performance.

To grow and protect the investment made in staff members, a learning organization pays careful attention to developing and retaining its people. Closely linked to development and retention of staff members are the importance of recognition and incentives for learning. Learning organizations ensure that time and effort spent on effective knowledge management and learning are recognized as core activities in the organization's time and performance management systems. Rewards for contributing to learning and knowledge development can be more conventional (e.g., career advancement, increased income, and greater formal status) or may be less conventional (e.g., informal peer status, time made available for study, or public acknowledgment for an innovative contribution made).

Learning organizations also provide a wide range of opportunities for individual and collective learning and development. Learning and development programs are available to ensure that individuals and teams develop the competencies of reflective practice and collaborative learning. While learning and development systems may focus on more formal programs, a learning organization is one where the maximum benefit is also leveraged from other learning opportunities such as day-to-day work experiences, team meetings, short-term secondments, and membership of task groups.

In a learning organization, an important source of individual learning and development is coaching and mentoring support from managers, specialists, and other experienced colleagues. High-quality coaching and mentoring can help reflective practice flourish. However, both involve skills that cannot be taken for granted and must be consciously developed in the organization. It cannot be assumed that good contract managers and technical specialists automatically make good coaches and mentors.

Learning organizations require and encourage the development of leadership competencies at all levels in the organizational hierarchy, not just at the top. Leadership is viewed as a valuable skill that is based on the possession of expertise and knowledge, not simply positional status.

\section{Knowledge}

Knowledge is a critical asset in every learning organization. Because learning is both a product of knowledge and its source, a learning organization recognizes that the two are inextricably linked and manages them accordingly.

The units of knowledge production are both the individual and the collective. Learning organizations understand that while knowledge is created in the minds of individuals, knowledge development thrives in a rich web of social contact among individuals, groups, and organizations. A learning organization provides creative opportunities for this knowledge to be developed and shared with others through interpersonal contact and access to documentation.

An organization's main repositories of knowledge are the design and delivery of its products and services and the strategies, systems, and procedures it has developed to guide its decision making. Learning organizations know how best to take a learning approach to the development of this embedded knowledge by putting in place the necessary systems and infrastructure for knowledge management. ${ }^{4}$

4 See, for example, ADB. 2008-. Knowledge Showcase. Manila: ADB. Available: www.adb.org/knowledgeshowcase/default.asp 
Tools, methods, and approaches available as of May 2009

Source: Asian Development Bank (ADB). 2008-. Knowledge Solutions. Manila: ADB. Available: www.adb.org/knowledgesolutions/default.asp

Feedback is the dynamic process of presenting and disseminating information to improve performance. Feedback mechanisms are increasingly being recognized as key elements of learning. Key (and often underutilized) sources of knowledge in organizations are the data and information that emerge from monitoring systems and the analyses, conclusions, and recommendations that arise from self- and independent evaluations. Learning organizations have sophisticated ways of designing evaluations with learning (as well as accountability) in mind. Methods such as after-action reviews and retrospects ${ }^{5}$ are successfully adopted and generate lessons that are carefully targeted at specific audiences. Learning organizations have systems

Knowledge is the true organ of sight, not the eyes.

-The Panchatantra that ensure that the outputs of self- and independent evaluations are made widely available, used to question orthodox thinking, and trigger creativity and innovation. Most significant changes are collected, systematically selected, and interpreted. ${ }^{6}$ Peer assists, ${ }^{7}$ drawing on individuals' expertise and documented lessons learned,

5 Organizational learning calls for nonstop assessment of performance - its successes and failures. This ensures that learning takes place and supports continuous improvement. After-action reviews and retrospects are tools that facilitate assessments by bringing together a team to discuss an activity or project openly and honestly.

6 The most significant change technique helps monitor and evaluate the performance of projects and programs. It involves the collection and systematic participatory interpretation of stories of significant change emanating from the field-stories about who did what, when, and why, and the reasons the event was important. It does not employ quantitative indicators.

7 Peer assists are events that bring individuals together to share their experiences, insights, and knowledge on an identified challenge or problem. They also promote collective learning and develop networks among those invited. 
are used in planning new initiatives to reduce the likelihood of repeated unintended negative outcomes. Action learning is used to tackle more intractable challenges. ${ }^{8}$

A learning organization recognizes the importance of a resilient organizational memory. Learning organizations ensure that individuals and teams are encouraged to use a range of ways of surfacing their tacit knowledge and making it available to others through carefully targeted documentation and collaborative working practices. Recognizing that organizations change in the direction in which they inquire, they leverage the powers of appreciative inquiry. ${ }^{9}$ Documentation is made accessible to others in the organization with a range of user-friendly information and communication technologies.

Learning organizations are networked with the wider world. They know how to create and run partnerships. ${ }^{10}$ Collaborative mutual learning arrangements with other organizations are common and fruitful.

\section{Technology}

Learning organizations know how to harness the power of information and communication technologies - without these technologies constraining knowledge management and learning. In a learning organization, information and communication technologies are used, among other purposes, to strengthen organizational identity; build and sustain learning communities; keep staff members, clients, and others informed and aware of corporate developments; create unexpected, helpful connections between people and

This is perhaps the most beautiful time in human history; it is really pregnant with all kinds of creative possibilities made possible by science and technology which now constitute the slave of man-if man is not enslaved by it. provide access to their knowledge and ideas; encourage innovation and creativity; share and learn from good practices $^{11}$ and unintended outcomes; strengthen relationships; develop and access organizational memory; share tools, methods, and approaches; celebrate successes; identify internal sources of expertise; and connect with the outside world.

The creative use of information and communication technologies such as shared document drives, intranet pages, online communities and networks, wikis and other collaborative work spaces, blogging and online storytelling, ${ }^{12}$ staff profile pages,$^{13}$ online webinars, podcasts, and social network analysis ${ }^{14}$ indicates that an organization takes learning seriously.

Finally, in a learning organization, sufficient opportunities are provided for staff members to learn how to make use of available information and communication technologies for knowledge management and learning. Box 2 offers a structured questionnaire with which to gauge perceptions of competencies to learn for change.

8 Action learning is a structured method that enables small groups to work regularly and collectively on complicated problems, take action, and learn as individuals and as a team while doing so.

9 Appreciative inquiry is the process of facilitating positive change in organizations. Its basic assumption is uncomplicated: every organization has something that works well. Appreciative inquiry is therefore an exciting generative approach to organizational development. At a higher level, it is also a way of being and seeing.

10 In development work as elsewhere, partnerships have a crucial role to play. To reach the critical mass required to reduce poverty, there must be more concerted effort, greater collaboration, alignment of inputs, and a leveraging of resources and effort. Understanding the drivers of success and the drivers of failure helps efforts to create and run them.

11 Good practice is a process or methodology that has been shown to be effective in one part of the organization and might be effective in another.

12 Storytelling is the use of stories or narratives as a communication tool to value, share, and capitalize on the knowledge of individuals.

13 Staff profile pages are dynamic, adaptive electronic directories that store information about the knowledge, skills, experience, and interests of people. They are a cornerstone of successful knowledge management and learning initiatives.

14 Power no longer resides exclusively (if at all) in states, institutions, or large corporations. It is located in the networks that structure society. Social network analysis seeks to understand networks and their participants and has two main focuses-the actors and their relationships in a specific social context. 


\section{Box 2. Seeking Feedback on Learning for Change ${ }^{15}$}

\section{Organization}

- There is an inspiring vision for learning and an organizational learning strategy that clearly communicates that learning is critical to organizational success.

- Leaders take an exemplary leading role in creating and sustaining a supportive learning culture.

- The formal organizational structure facilitates learning, adaptation, and change.

- Sanctioned informal organizational structures enable and encourage learning across formal structural boundaries.

- Good use is made of communication systems to facilitate the lateral transfer of information and knowledge and to minimize the development of "silos."

- Adequate resources are allocated for learning in terms of time allocation, specialist support staff, budgets for knowledge management infrastructure, formal and informal communities of practice and other value networks, and learning and development programs.

- A balanced approach to learning that recognizes the importance of both planned and emergent learning is taken.

- Planned learning is addressed through the careful design of strategy, structure, systems, procedures, and plans.

- Emergent learning is encouraged by creating opportunities for informal sharing of knowledge and experience.

- Failures and unintended outcomes are the focus of constructive discussions leading to new approaches. When such incidents involve clients, care is taken to protect their reputation.

\section{People}

- Staff members are required to be reflective practitioners to reflect on their experience, develop experience-based theories of change, continuously test these in practice with colleagues, and use their understanding and initiative to contribute to knowledge development.

- All staff members make frequent use of a range of tools, methods, and approaches for learning and collaborating with others.

- Staff members experience a high level of psychological safety and trust; they can rely on colleagues and are not exposed to unfair negative criticism.

- Teams operate as learning communities in which success and unexpected outcomes are analyzed and in which sensitively expressed dissent, conflict, and debate are encouraged as positive sources of learning.

- Staff members are encouraged to look outside the organization for new ideas, trends, and practices and to share what they learn with colleagues.

- Equal attention is paid to developing and retaining staff members at all levels.

- Staff members successfully use a wide range of opportunities for individual and team-based learning and development.

- Time and effort spent by staff members on learning and knowledge development are recognized as core activities in the organization's time and performance management systems.

- A wide range of formal and informal rewards and incentives for contributing to organizational learning and knowledge development is used (e.g., career advancement, increased income, informal peer status, additional time provided for study, and public acknowledgment for innovative contributions made).

- Leadership (based on the possession of expertise and knowledge) is expected from staff members at all levels in the organizational hierarchy.

\section{Knowledge}

- There is a widespread recognition that while knowledge is created in the minds of individuals, knowledge development thrives in a rich web of professional networks among individuals.

- Important knowledge is easily accessible to people who need and use it.

- There are creative opportunities for knowledge to be developed and shared with others by facilitating networks between individuals.

- The design and delivery of products and services demonstrate how effective the organization is at applying what it has learned about the nature of good practice.

- The necessary systems and infrastructure for knowledge management are in place, understood, and working effectively.

- Evaluations are carefully designed with learning (as well as accountability) in mind. Systems ensure that the outputs of internal and independent evaluations are made widely available; carefully examined; and used to influence decision making and planning, question orthodox thinking, and trigger creativity and innovation.

- Peer assists, drawing on individuals' expertise and documented lessons learned, are used in planning new initiatives to reduce the likelihood of repeated and unintended negative outcomes.

- The organization has a resilient organizational memory and is not vulnerable to the loss of important knowledge when staff members move to other jobs in the organization or leave.

- Individuals and teams successfully use a range of methods for surfacing their tacit knowledge and making it available to others, for example, by using carefully targeted documentation and collaborative working practices.

- Adoption of after-action reviews and retrospects to learn from experience has been successful.

\footnotetext{
15 The use of a six-point scale from Strongly Agree to Strongly Disagree is recommended.
} 
Technology

- There is a thorough and shared understanding of the value of information and communication technologies for knowledge management and learning.

- Information and communication technologies facilitate but do not drive or constrain knowledge management and learning in the organization.

- Information and communication technologies are successfully used to create and sustain learning communities.

- Information and communication technologies are successfully used to keep people informed and aware of corporate developments.

- Information and communication technologies are successfully used to create unexpected, helpful connections between people and to provide access to their knowledge and ideas.

- Information and communication technologies are successfully used to encourage innovation and creativity.

- Information and communication technologies are successfully used to enable people to share and learn from good practices and unintended outcomes.

- Information and communication technologies are successfully used to enable people to identify internal sources of expertise.

- Creative use of information and communication technologies is high. At least five of the following have been successfully adopted: shared document drives, intranet pages, online communities and networks, wikis and other means of collaborative document production, blogging, online storytelling, lessons learned databases, staff profile pages, online webinars, podcasts, and social network mapping.

- Sufficient opportunities are provided for staff members to learn how to make use of available information and communication technologies for learning and knowledge sharing.

\section{Further Reading}

ADB. 2008-. Compendium of Knowledge Solutions. Manila. Available: www.adb.org/documents/information/ knowledge-solutions/knowledge-solutions-1-38.pdf

- 2009a. Overcoming Roadblocks to Learning. Manila. Available: www.adb.org/documents/

information/knowledge-solutions/overcoming-roadblocks-to-learning.pdf

- 2009b. Dimensions of the Learning Organization. Manila. ADB. Available: www.adb.org/documents/ information/knowledge-solutions/dimensions-learning-organization.pdf

-2009c. Learning for Change in ADB. Manila. ADB. Available: www.adb.org/documents/books/learningfor-change/default.asp

\section{For further information}

Contact Olivier Serrat, Head of the Knowledge Management Center, Regional and Sustainable Development Department, Asian Development Bank (oserrat@adb.org). 


\section{Kolutions}

Asian Development Bank

$A D B$ 's vision is an Asia and Pacific region free of poverty. Its mission is to help its developing member countries reduce poverty and improve the quality of life of their people. Despite the region's many successes, it remains home to two thirds of the world's poor: 1.8 billion people who live on less than $\$ 2$ a day, with 903 million struggling on less than $\$ 1.25$ a day. ADB is committed to reducing poverty through inclusive economic growth, environmentally sustainable growth, and regional integration.

Based in Manila, ADB is owned by 67 members, including 48 from the region. Its main instruments for helping its developing member countries are policy dialogue, loans, equity investments, guarantees, grants, and technical assistance.

Knowledge Solutions are handy, quick reference guides to tools, methods, and approaches that propel development forward and enhance its effects. They are offered as resources to ADB staff. They may also appeal to the development community and people having interest in knowledge and learning.

The views expressed in this publication are those of the author and do not necessarily reflect the views and policies of the Asian Development Bank (ADB) or its Board of Governors or the governments they represent. $A D B$ encourages printing or copying information exclusively for personal and noncommercial use with proper acknowledgment of ADB. Users are restricted from reselling, redistributing, or creating derivative works for commercial purposes without the express, written consent of ADB.

Asian Development Bank

6 ADB Avenue, Mandaluyong City

1550 Metro Manila, Philippines

Tel +6326324444

Fax +6326362444

knowledge@adb.org

www.adb.org/knowledgesolutions 